Der Große Reuter

Springer Universalwörterbuch Medizin, Pharmakologie und Zahnmedizin 
P. Reuter

\section{Der Große Reuter}

\section{Springer Universalwörterbuch Medizin, Pharmakologie und Zahnmedizin}

Englisch-Deutsch

Springer 
Peter Reuter, Dr. med.

Fort Myers

Florida, USA

reutermedical@comcast.net

\section{ISBN 3-540-25102-2 Springer Berlin Heidelberg New York}

Bibliografische Information Der Deutschen Bibliothek

Die Deutsche Bibliothek verzeichnet diese Publikation in der Deutschen Nationalbibliografie; detaillierte bibliografische Daten sind im Internet unter http://dnb.ddb.de abrufbar

Dieses Werk ist urheberrechtlich geschützt. Die dadurch begründeten Rechte, insbesondere die der Übersetzung, des Nachdrucks, des Vortrags, der Entnahme von Abbildungen und Tabellen, der Funksendung, der Mikroverfilmung oder der Vervielfältigung auf anderen Wegen und der Speicherung in Datenverarbeitungsanlagen, bleiben, auch bei nur auszugsweiser Verwertung, vorbehalten. Eine Vervielfältigung dieses Werkes oder von Teilen dieses Werkes ist auch im Einzelfall nur in den Grenzen der gesetzlichen Bestimmungen des Urheberrechtsgesetzes der Bundesrepublik Deutschland vom 9. September 1965 in der jeweils geltenden Fassung zulässig. Sie ist grundsätzlich vergütungspflichtig. Zuwiderhandlungen unterliegen den Strafbestimmungen des Urheberrechtsgesetzes.

\section{Springer ist ein Unternehmen von Springer Science+Business Media}

springer.de

(C) Springer-Verlag Berlin Heidelberg 2005

Printed in Germany

Die Wiedergabe von Gebrauchsnamen, Warenbezeichnungen usw. in diesem Werk berechtigt auch ohne besondere Kennzeichnung nicht zu der Annahme, dass solche Namen im Sinne der Warenzeichen- und Markenschutzgesetzgebung als frei zu betrachten wären und daher von jedermann benutzt werden dürften.

Produkthaftung: Für Angaben über Dosierungsanweisungen und Applikationsformen kann vom Verlag keine Gewähr übernommen werden. Derartige Angaben müssen vom Anwender im Einzelfall anhand anderer Literaturstellen auf ihre Richtigkeit überprüft werden.

Planung: Thomas Mager, Heidelberg

Redaktion: Sylvia Blago, Heidelberg

Herstellung: Frank Krabbes, Heidelberg

Umschlaggestaltung: KünkelLopka $\mathrm{GmbH}$, Heidelberg

Satz: wiskom e.K., Friedrichshafen

Gedruckt auf säurefreiem Papier

SPIN: 11401216

$14 / 2109 f k-543210$ 
Unser Ziel war es, ein Werk zu kompilieren, dass sowohl Benutzern aus dem Bereich der Medizin und der Zahnmedizin, als auch Übersetzern ein umfangreiches Vokabular anbietet. Mit rund 140.000 Hauptund Untereinträgen, Anwendungsbeispielen, Abkürzungen und Akronymen aus der Medizin, Zahnmedizin, Pharmazie und Pharmakologie und mehr als 400.000 Übersetzungen, bietet das Werk ein Vokabular, das sowohl in Klinik und Praxis als auch Studium, Lehre und Forschung von Bedeutung ist.

Auch wenn der größte Teil der Stichwörter aus der amerikanischen Literatur stammt, haben wir doch britische Varianten aufgenommen, wo immer dies inhaltlich sinnvoll war.

Der Anhang enthält Tabellen für „Maße und Gewichte“, „Umrechnungstabellen für Temperaturen“, „Normalwerte wichtiger Laborparameter" sowie 14 anatomische Tafeln.

Leider konnten nicht alle Fachtermini erfasst werden und uns ist auch bewusst, dass das Material nicht fehlerfrei ist. Deshalb die Bitte an alle Benutzer des Werkes um positives Feedback, damit wir in der 2. Auflage Verbesserungen machen können.

Ich bedanke mich bei allen Verlagsmitarbeitern für ihre unermüdliche Hilfe und Unterstützung.

Fort Myers, im Mai 2005

Für ihre Mithilfe sei besonders gedankt:

Dr. Sylvia Blago

Dr. Thomas Mager

Dr. Claus Puhlmann
It was our intention to compile a dictionary that provides users from the medical and dental field as well as translators with a comprehensive vocabulary. With some 140,000 entries, subentries, illustrative phrases, abbreviations and acronyms from medicine, dentistry, pharmaceutics and pharmacology and more than 400,000 translations the dictionary provides a vocabulary that covers work in the hospital and in the office, but also medical and dental studies as well as teaching and research.

Although most entries are American English, we also included British terms whenever necessary or reasonable.

The appendix contains tables for "Weights and Measures", "Conversion Tables for Temperatures", "Laboratory Reference Range Values" as well as 14 anatomical plates.

It was impossible to include all relevant entries and we are aware that there are some mistakes and errors. Therefore, we are asking for your positive feedback to help us make improvements in the second edition.

I would like to thank everybody involved at Springer for their continuing help and support.

Peter Reuter

Special thanks to: 


\section{Inhaltsverzeichnis/Table of Contents}

\section{Hinweise zur Benutzung/}

Guide to the Dictionary.

Hinweise zur Benutzung der Lautschrift/

A Guide to Pronunciation

\section{Abkürzungsverzeichnis/}

List of Abbreviations.

Lexikonteil/

A-Z Vocabulary.

Anhang/

Appendix

Maße und Gewichte/

Weights and Measures

Umrechnungstabellen für Temperaturen/

Conversion Tables for Temperatures

Normalwerte wichtiger Laborparameter/

Laboratory Reference Range Values . .

Anatomische Tafeln/

Anatomical Plates. 


\section{Hinweise zur Benutzung}

Hauptstichwörter werden auf der Grundlage eines Buchstaben-für-Buchstaben-Systems eingeordnet. Bei mehrsilbigen Stichwörtern [Ausnahme: Komposita] wird die Silbentrennung angezeigt.

Umlaute werden bei der Alphabetisierung nicht berücksichtigt, d.h., ä, ö, ü werden als a, o bzw. u eingeordnet. Kursive Vorsilben, numerische und chemische Präfixe sowie griechische Buchstaben werden ebenfalls nicht beachtet.

Mehrworteinträge erscheinen in der Regel als Untereinträge $\mathrm{zu}$ einem logischen Überbegriff. Untereinträge werden genauso wie Hauptstichwörter alphabetisch eingeordnet. Die Pluralform wird bei der Einordnung nicht berücksichtigt. Das Gleiche gilt für Präpositionen, Konjunktionen und Artikel.

Vier Schriftarten werden zur Gliederung der Einträge eingesetzt:

Halbfett für den Haupteintrag

Auszeichnungsschrift für Untereinträge, Anwendungsbeispiele und Redewendungen

Grundschrift für die Übersetzung(en)

Kursiv für bestimmende Zusätze, Sachgebietsangaben und Verweise

\section{Unterteilung der Stichwortartikel}

Hat das Stichwort mehrere grammatische Bedeutungen, werden die einzelnen Wortarten durch römische Ziffern unterschieden.

Arabische Ziffern werden zur Unterscheidung der verschiedenen Bedeutungsfacetten eingesetzt. Ihre fortlaufende Nummerierung ist unabhängig von den oben genannten römischen Ziffern.

\section{Wortarten \\ Haupteinträge haben eine kursive Wortartangabe [siehe auch „Abkürzungsverzeichnis“]. \\ Gehört ein Haupteintrag mehreren grammatikali- schen Kategorien an, steht die entsprechende Wort- artbezeichnung unmittelbar hinter jeder römischen Ziffer.}

\section{Verweise}

Verweise werden durch Pfeile $[\rightarrow]$ gekennzeichnet.

\section{Guide to the Dictionary}

Main entries are alphabetized using a letter-for-letter system. For entries of more than one syllable syllabification is given. However, this does not apply to compound entries.

Umlauts are ignored in alphabetization and ä, ö, ü are treated as a, o, u, respectively. Italic and chemical prefixes, numbers, and Greek letters are ignored in alphabetization.

As a rule multiple-word terms are given as subentries under the appropriate main entry. They are alphabetized letter by letter just like the main entries. Plural forms, prepositions, conjunctions, and articles are always disregarded in alphabetization of subentries.

Four styles of type are used for different categories of information:

boldface for the main entry

lightface for subentries, illustrative phrases and idiomatic expressions

plainface for the translation(s)

italic for restrictive labels, subspecialties and crossreferences

\section{Subdivision of Entries}

If the entry word is used in more than one grammatical form, Roman numerals are used to distinguish the various parts of speech.

Arabic numerals are used to distinguish the various meanings of the entry. This consecutive numbering is used regardless of the Roman numerals mentioned above.

\section{Parts of Speech}

Main entries have an italicized part-of-speech label [see also "List of Abbreviations"].

If the entry word is used in more than one grammatical form the appropriate part-of-speech label is given immediately after every Roman numeral.

\section{Cross-references}

Cross-references are indicated by arrows $[\rightarrow]$. 


\section{Hinweise zur Benutzung der Lautschrift \\ A Guide to Pronunciation}

\section{Lautschriftsymbole und Betonungsakzente}

Die in diesem Wörterbuch angegebenen Aussprachen benutzen die Zeichen der „International Phonetic Association (IPA)“.

['] zeigt den Hauptakzent an. Die auf das Zeichen folgende Silbe wird stärker betont als die anderen Silben des Wortes.

[1] zeigt den Nebenakzent an. Eine Silbe, die mit diesem Symbol gekennzeichnet ist, wird stärker betont als nicht markierte Silben aber schwächer als mit einem Hauptakzent markierte Silben.

\section{Vokale und Diphthonge}

Die lange Betonung eines Vokals wird durch [:] angezeigt.

\begin{tabular}{|c|c|c|}
\hline$[æ]$ & hat & [hæt] \\
\hline [e] & red & [red] \\
\hline [eI] & rain & [reIn] \\
\hline [a] & got & [gat] \\
\hline [a:] & car & [ca:r] \\
\hline [eə] & chair & [t ferr] \\
\hline [i:] & key & {$[\mathrm{kiz}]$} \\
\hline I & in & [In] \\
\hline [Іа & fear & [fiər] \\
\hline & eye & [aI] \\
\hline
\end{tabular}

Konsonanten

Die Verwendung der Konsonanten [b] [d] [g] [h] [k] [l] [m] [n] [p] [t] ist im Deutschen und Englischen gleich.

$\begin{array}{lll}{[\mathrm{r}]} & \text { arm } & \text { [a:rm] } \\ {[\mathrm{s}]} & \text { salt } & \text { [so:lt] } \\ {[\mathrm{v}]} & \text { vein } & \text { [vern] } \\ {[\mathrm{w}]} & \text { wave } & \text { [werv] } \\ {[\mathrm{z}]} & \text { zoom } & \text { [zu:m] } \\ {\left[\mathrm{t} \int\right]} & \text { chief } & \left.\text { [t } \int \mathrm{i}: \mathrm{f}\right] \\ {[\mathrm{j}]} & \text { yoke } & \text { [jouk] }\end{array}$

\author{
Phonetic Symbols and Stress Marks
}

The pronunciation in this dictionary is indicated by the alphabet of the "International Phonetic Association (IPA)".

['] indicates primary stress. The syllable following it is pronounced with greater prominence than other syllables in the word.

$\left[{ }_{1}\right]$ indicates secondary stress. A syllable marked for secondary stress is pronounced with greater prominence than those bearing no stress mark at all but with less prominence than syllables marked for primary stress.

\section{Vowels and Diphthongs}

The long pronunciation of a vowel is indicated by [ $\mathrm{l}]$.

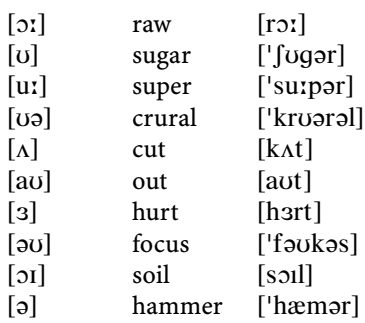

\section{Consonants}

The use of the consonants [b] [d] [g] [h] [k] [l] [m] [n] [p] [t] is the same in English and German pronunciation.

$\begin{array}{lll}{\left[\mathrm{d}_{3}\right]} & \text { bridge } & {[\mathrm{brid} 3]} \\ {[\mathrm{y}]} & \text { pink } & {[\mathrm{pInk}]} \\ {\left[\int\right]} & \text { shin } & {\left[\int \mathrm{In}\right]} \\ {[3]} & \text { vision } & {[\text { 'vizn }]} \\ {[\theta]} & \text { throat } & {[\theta \text { rout }]} \\ {[ð]} & \text { there } & {[\text { ðeər] }} \\ {[\mathrm{x}]} & \text { loch } & {[\mathrm{lax}]}\end{array}$


Zusätzliche Symbole für Stichwörter aus anderen Sprachen

\begin{tabular}{|c|c|c|c|c|}
\hline [a] & natif & [na'tıf] & Backe & [bakə] \\
\hline [ع] & lettre & ['letrə] & Bett & {$[b c t]$} \\
\hline [i] & iris & [i'ris] & Titan & [ti'ta:n] \\
\hline [o] & dos & [do] & Hotel & [ho'tel] \\
\hline [y] & dureé & [dy're] & mürbe & ['myrbə] \\
\hline [0] & note & {$[\mathrm{not}]$} & toll & [tol] \\
\hline$[\mathrm{u}]$ & nourrir & [nu'risr] & mutieren & [mu'tirrən] \\
\hline [œ] & neuf & [nœf] & Mörser & ['mœrzər] \\
\hline[] & cuisse & [kuis] & & \\
\hline [ø] & feu & [fø] & Ödem & [ø'de:m] \\
\hline [n] & baigner & [bc'ne] & & \\
\hline [œj] & feuille & [fœj] & & \\
\hline$[a: j]$ & tenailles & [to'na:j] & & \\
\hline [ij] & cochenille & [kof'nij] & & \\
\hline$\varepsilon j]$ & sommeil & [so'mej] & & \\
\hline aj] & maille & [maj] & & \\
\hline 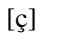 & & & Becher & ['beçər] \\
\hline
\end{tabular}

Additional Symbols used for Entries from other Languages 


\section{Abkürzungsverzeichnis/List of Abbreviations}

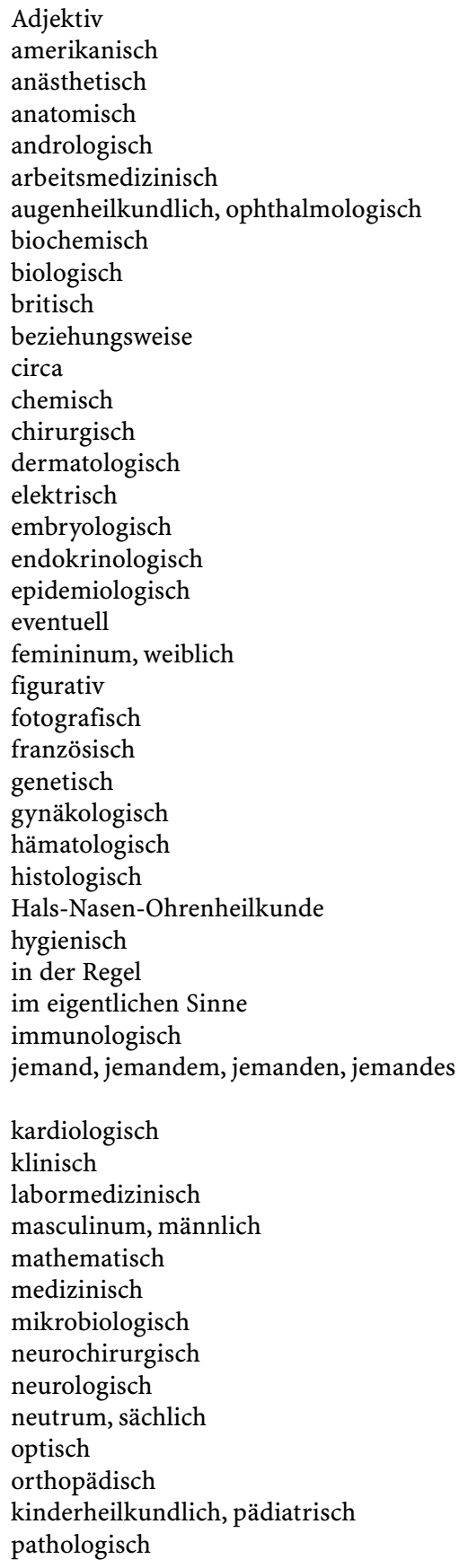

\begin{tabular}{|c|c|}
\hline adj & adjective \\
\hline ameri. & American \\
\hline anästh. & anesthetic \\
\hline anatom. & anatomical \\
\hline androl. & andrologic \\
\hline arbeitsmed. & occupational medical \\
\hline augenheil. & ophthalmologic \\
\hline biochem. & biochemical \\
\hline biolog. & biological \\
\hline brit. & British \\
\hline bzw. & respectively, or \\
\hline ca. & approximately \\
\hline chem. & chemical \\
\hline chirurg. & surgical \\
\hline dermatol. & dermatologic \\
\hline elektr. & electric \\
\hline embryolog. & embryologic \\
\hline endokrin. & endocrinologic \\
\hline epidemiol. & epidemiologic \\
\hline evtl. & possibly, perhaps \\
\hline $\mathrm{f}$ & feminine, female \\
\hline fig. & figurative(ly) \\
\hline foto. & photographic \\
\hline franz. & French \\
\hline genet. & genetic \\
\hline gynäkol. & gynecologic \\
\hline hämat. & hematologic \\
\hline histolog. & histologic \\
\hline HNO & ear, nose and throat (ENT) \\
\hline hygien. & hygienic \\
\hline i.d.R. & as a rule \\
\hline i.e.S. & in a narrower sense, in the true sense \\
\hline immunolog. & immunologic \\
\hline jd., jdm., jdn., jds. & $\begin{array}{l}\text { someone, to someone, someone, of } \\
\text { someone }\end{array}$ \\
\hline kardiol. & cardiologic \\
\hline klin. & clinical \\
\hline labor. & laboratory \\
\hline $\mathrm{m}$ & masculine, male \\
\hline mathemat. & mathematical \\
\hline medizin. & medical \\
\hline mikrobiolog. & microbiological \\
\hline neurochirurg. & neurosurgical \\
\hline neurol. & neurologic \\
\hline nt & neuter \\
\hline opt. & optical \\
\hline orthopäd. & orthopedic \\
\hline pädiat. & pediatric \\
\hline patholog. & pathologic \\
\hline
\end{tabular}

adj

anästh

androl.

arbeitsmed.

augenheil.

biochem.

biolog

brit.

bzw.

chirurg.

embryolog.

endokrin

epidemiol

evtl

franz.

genet.

hämat.

histolog

$\mathrm{HNO}$

hygien.

i.d.R

immunolog

jd., jdm., jdn., jds.

kardiol.

klin.

labor.

medizin.

mikrobiolog

neurochirurg.

nt

pädiat.

patholog. adjective

anatomical

andrologic

ophthalmologic

biochemical

biological

British

respectively, or

approximately

dermatologic

endocrinologic

epidemiologic

possibly, perhaps

feminine, female

French

gynecologic

hematologic

histologic

ear, nose and throat (ENT)

hygienic

in a narrower sense, in the true sense mmunologic

someone

laboratory

masculine, male

mathematical

medical

neurologic

optical

pathologic 


\author{
pharmakologisch \\ physikalisch \\ physiologisch \\ Plural, Mehrzahl \\ Präfix, Vorsilbe \\ psychiatrisch \\ psychologisch \\ pulmonologisch \\ radiologisch \\ rechtsmedizinisch \\ sich \\ siehe unter \\ so genannt \\ soziologisch \\ sportmedizinisch \\ statistisch \\ Suffix, Nachsilbe \\ technisch \\ unter anderem; und andere \\ und Ähnliche(s) \\ unter Umständen \\ urologisch \\ und so weiter \\ Verb \\ vor allem \\ intransitives Verb \\ transitives Verb \\ zum Beispiel \\ zum Teil \\ zahnmedizinisch
}

pharmakol.

physik.

physiolog.

$\mathrm{pl}$

präf.

psychiat.

psychol.

pulmonolog.

radiolog.

rechtsmed.

s.

s.u.

sog.

soziol.

sportmed.

statist.

suf.

techn.

u.a.

u.ä., u.Ä.

u.U.

urolog.

usw.

$\mathrm{v}$

v.a.

vi

$\mathrm{vt}$

z.B.

z.T.

zahnmed. pharmacologic

physical

physiologic

plural

prefix

psychiatric

psychologic

pulmonologic

radiologic

forensic

oneself

see under

so called

sociologic

sports medical

statistical

suffix

technical

among others; and others

and similar

possibly, perhaps

urologic

and so forth

verb

especially

intransitive verb

transitive verb

for example

partially

dental 